553

\section{FIRST-IN-CLASS INHIBITORS OF ERAP1 ALTER THE IMMUNOPEPTIDOME OF CANCER, DRIVING A DIFFERENTIATED T CELL RESPONSE LEADING TO TUMOR GROWTH INHIBITION}

${ }^{1}$ Peter Joyce ${ }^{*},{ }^{2}$ Martin Quibell, ${ }^{3}$ Jason Shiers, ${ }^{3}$ Carmen Tong, ${ }^{3}$ Kristopher Clark, ${ }^{4}$ Nicola Ternette, ${ }^{2}$ Kate Anderton, ${ }^{2}$ Jessica Sette, ${ }^{4}$ Wayne Paes, ${ }^{2}$ Andrew Leishman. ${ }^{1}$ Grey Wolf Therapeutics Ltd, Oxford, UK; ${ }^{2}$ Grey Wolf Therapeutics, Oxford, UK; ${ }^{3}$ Sygnature Discovery, Nottingham, UK; ${ }^{4}$ University of Oxford, Oxford, UK

Background Clinical data demonstrates increased antigen presentation diversity is an important factor in determining response rates to checkpoint inhibitors. ${ }^{1}$ In addition to tumor mutational burden, increased HLA heterozygosity and HLA evolutionary diversity are non-overlapping factors which further diversify the immunopeptidome and improve clinical response to checkpoint therapies. ${ }^{2}{ }^{3}$ Endoplasmic reticulum aminopeptidase 1 (ERAP1) is an enzyme that trims peptides loaded into classical and nonclassical MHC Class I molecules. ${ }^{4}$ 5 Ablation of mouse ERAAP modifies the immunopeptidome, resulting in improved immunogenicity, generation of CD8 $+\mathrm{T}$ cell responses and tumor growth inhibition. ${ }^{6} 7$ We report the characterisation of ERAP1 inhibitors in syngeneic tumor models and development of biomarkers to enable translation of this mechanism into the clinic.

Methods Human and mouse cancer cell lines treated with ERAP1 inhibitors were assessed by immunopeptidomics ${ }^{8}$ to profile peptide repertoire changes. ERAP1 inhibitor with and without checkpoint inhibition were used to treat syngeneic mouse tumor models, followed by analysing effects on the $\mathrm{T}$ cell receptor (TCR) repertoire, RNA sequencing profile, immune cell infiltration and tumor growth inhibition.

Results Extensive analysis of the immunopeptidomes of diverse cancer cell lines robustly show that ERAP1 inhibition modulates the cancer-related antigen repertoire across diverse ERAP1 and HLA genotypes and cancer-type backgrounds. ERAP1 inhibition drives changes in $\mathrm{T}$ cell activation and response, leading to increased $\mathrm{T}$ cell infiltration into CT26 syngeneic tumors and alteration of the TCR repertoire at early and late timepoints in tumor growth. Consistent peptide length changes in the immunopeptidome, caused by ERAP1 inhibition, is a proof of mechanism biomarker, whilst tumor immunohistochemisty, TCR repertoire analysis and RNA sequencing are potential proof of principle biomarkers that can all be translated into the clinic. Importantly, the antigen and $\mathrm{T}$ cell changes we see following ERAP1 inhibition lead to robust tumor growth inhibition in different syngeneic mouse models when combined with anti-PD-1. We are also exploring the potential of ERAP1 inhibitors to enhance tumour immune responses in combination with additional therapies (e.g. chemotherapy and radiotherapy), across different tumor microenvironments.

Conclusions Grey Wolf Therapeutics ERAP1 inhibitors significantly modify the immunopeptidome and combination with anti PD-1 leads to significant TCR repertoire change, $\mathrm{T}$ cell infiltration and tumor growth inhibition in syngeneic mouse tumor models. These data provide the foundation from which we will explore the potential of our first-in-class ERAP1 inhibitor development candidate in the clinic, as well as identifying useful biomarkers to demonstrate desired biological activity.

\section{REFERENCES}

1. Rizvi N, Hellmann MD, Snyder A, et al. Mutational landscape determines sensitivity to PD-1 blockade in non-small cell lung cancer. Science 2015;348 (6230):124-128.
2. Chowell D, uc G T Morris LGT,2 3, Grigg CM, et al Patient HLA class I genotype influences cancer response to checkpoint blockade immunotherapy. Science 2018;359 (6375):582-587.

3. Chowell D, Chirag Krishna, Federica Pierini, et al Evolutionary divergence of HLA class I genotype impacts efficacy of cancer immunotherapy. Nature Medicine 2019;25(11):1715-1720.

4. Shastri $\mathrm{N}$, Nagarajan $\mathrm{N}$, Lind $\mathrm{KC}$, et al. Monitoring peptide processing for $\mathrm{MHC}$ class I molecules in the endoplasmic reticulum. Curr Opin Immunol 2014;26:123127.

5. Mpakali A, Maben Z, Stern LI, et al. Molecular pathways for antigenic peptide generation by ER aminopeptidase 1. Mol Immunol 2018:13:50-57.

6. James E, Bailey I, Sugiyarto G, et al. Induction of protective antitumor immunity through attenuation of ERAAP function. J Immunol 2013;190(11):5839-5846.

7. Manguso RT, Pope, HW, MD Zimmer, et al In vivo CRISPR screening identifies Ptpn2 as a cancer immunotherapy target. Nature 2017;547(7664):413-418.

8. Purcell AW, Ramarathinam SH, Ternette N. Mass spectrometry-based identification of MHC-bound peptides for immunopeptidomics. Nat Protoc 2019;14 (6):1687-1707.

http://dx.doi.org/10.1136/jitc-2021-SITC2021.553 